\title{
Utilisation de la crue au maintien de la hauteur de chute de l'usine de Faux-la-Montagne
}

\section{The use of floods for head maintenance at the Faux-la-Montagne power plant}

\author{
PAR G. MARQUENET \\ Ingkeneur aU Laboratome Natronal d'HydraUligue
}

English synopsis p. 218

L'usine de Faux-la-Montagne (aménagement de la Vienne et de la Maulde, a l'amont de Peyrat-le-Château) est une petite usine automatique de $3000 \mathrm{~kW}$.

Les terrains à l'aval de l'usine sont marécageux; le plan d'eau de la Maulde dont le lit est incertain, est mal connu en période de crue.

La Direction Régionale de l'Equipement Hydraulique, Massif Central I, craignait, qu'en période de hautes eaux, l'usine se trouve noyée par la montée du plan d'eau aval; pour abaisser le plan d'eau à l'aval de l'usine, elle a été amenée à projeter la jonction du canal de fuite au canal de déversement à forte pente, dans le but de provoquer par écoulement à grande vitesse des eaux en excès dans celui-ci, un effet d'injection à l'extrémité du canal de fuite. La jonction de ces deux canaux, à une soixantaine de mètres en aval de l'usine, s'effectue en plan sous un angle de $26^{\circ}$ environ.

Pour vérifier l'efficacité de ce dispositif, une étude sur modèle au $1 / 25$ a été effectuce au Laboratoire National d'Hydraulique à Chatou. Nous avons cherché, non seulement à assurer un fonctionnement régulier de l'usine automatique par un abaissement suffisant du plan d'eau aval, mais également à maintenir constante et maximum la hauteur de chute de l'usine, en période de crue. L'idée de base a été d'utiliser au mieux le canal de déversement comme injecteur à l'extrémité aval du canal de fuite.
C'était ainsi reprendre l'idée des renforceurs de chute; c'est sous cet angle que nous examinerons dans cette note le cas particulier de Fauxla-Montagne.

Nous verrons que le gain relatif, dans ce cas d'espèce, est faible (quelques pour cent). L'intérêt des renforceurs de chute en général n'est pas mis en cause; mais les caractéristiques de cette usine de moyenne chute : hauteur de chute $35,50 \mathrm{~m}$, débit turbiné : $8 \mathrm{~m}^{3} / \mathrm{s}$, crue exceptionnelle : $20 \mathrm{~m}^{3} / \mathrm{s}$, ne permettaient pas d'espérer davantage. Il était cependant intéressant, sans augmenter le prix de construction, de chercher à utiliser au mieux l'énergie de l'eau non turbinće.

Lidée de maintenir à sa valeur normale la hauteur de chute d'une usine de basse chute en particulier, a fait l'objet de quelques réalisations au début du siècle. Dans l'ensemble, le coût supplémentaire de la construction, qu'entrainaient ces dispositifs, rendait l'opération peu économique. Il semble que les nouvelles conceptions des usines de basse chute (usines piles, usines submersibles) permettent d'obtenir pour ces usines des' résultats plus satisfaisants.

C'est pourquoi nous croyons intéressant d'examiner successivement quelques généralités sur les renforceurs de chute, le cas particulier de Faux-la-Montagne, et les conceptions nouvelles en matière d'usines de basse chute. 
CHAPITRE I

\section{GENERALITES SUR LES RENFORCEURS DE CHUTE}

\section{I. - Principe}

On sait que la puissance des usines de basse chute diminue en période de crue. Le plan d'eau à l'amont de l'usine est, en général, maintenu à une cote sensiblement constante et voisine de la cote maximum admissible, tandis que le plan d'eau aval s'élève avec la crue. La hauteur de chute brute de l'usine diminue en période de hautes caux, et parfois dans des proportions extrêmement importantes. Par exemple, pour l'usine aménagée sur le bief de Kachlet, sur le Danube près de Passau, la hauteur de chute tomberait de $9 \mathrm{~m}$ à $0,5 \mathrm{~m}$ (débit moyen annuel $700 \mathrm{~m}: \mathrm{s}$, débit en période de hautes eaux $\left.6000 \mathrm{~m}^{3} / \mathrm{s}\right)(\mathrm{l})$.

En première approximation, la puissance de l'usine varie comme la puissance $3 / 2$ de la hauteur de chute, en négligeant en particulier la variation du rendement de la turbine avec la charge. On a, en effet, en désignant par :

$P$ la puissance de la turbine,

$Q$ le débit turbiné,

H la hauteur de chute de l'usine,

$$
\mathrm{P}=k \mathrm{QH}\left(\operatorname{avec} \mathrm{Q} \# k^{\prime} \mathrm{H}^{1 / 2}\right)
$$

soit :

$$
\mathrm{P}=k^{\prime \prime} \mathrm{H}^{3 / 2}
$$

1. Economiquement, on ne peut envisager l'installation de turbines supplémentaires destinées à fonctionner exclusivement au moment des crues.

D'autre part, la surouverture d'une turbine est limitée, elle conduit d'ailleurs à un rendement plus faible.

2. Il faul plutôt chercher à récupérer une partie de ia hatuteur de chute ainsi perdue, en utilisant l'energie de l'eau en excès pour abaisser le plan d'eau aval immédiatement à la sortie de la turbine, ou accrôttre la dépression dans le diffuseur de la turbine.

Différents dispositifs ont été successivement proposés sous te nom, d'ailleurs impropre, de renforceurs de chute. Nous allons examiner les plus intéressants.

\section{II. - Dispositifs proposés}

Quelques réalisations sont à retenir : l'usine de Chèvres près de Genève qui a été pourvue par SAUgeY d'une vanne jouant le ròle d'injecteur, quelques installations aux Etats-Unis équipées de renforceurs d'Henschels et de tuyères de Freman, et l'usine du Mitchelldam de l'Alabama Power Company.

\section{$1^{\circ}$ Renforceurs d'Herschell et de Freeman.}

Les dispositifs d'Herschell et de Freman agissent directement sur le diffuseur de la turbine.

a) Le renforceur d'Hrrschele (1907) conçu pour l'usine de la Plaine à Genève se présente

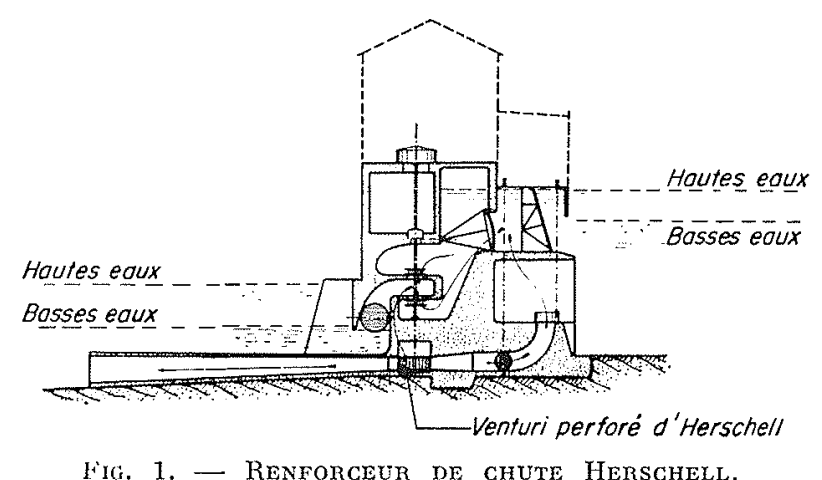

Fig. 1. - Renforceur de chute Herschell. Usine de la Plaine (Genève).

sous l'aspect d'un Venturi de grande dimension, dont le col perforé peut être mis en communication avec la chambre de décharge de la turbine (fig. 1) (II). Les eaux en excès sont évacuées par ce Venturi et provoquent une dépression dans la chambre de décharge. Le rendement du dispositif défini par le quotient :

$$
\text { énergie récupérée }
$$

énergie de l'eau passant par le renforceur varie de 12 à $20 \%$.

L'effet du renforceur ne commence guère à se faire sentir que lorsque le débit évacué par celuici est le double du débit turbiné. Des essais efrectués par Duri semblent montrer que le gain 
de chute décroît à peu près linéairement lorsque croit le rapport :

\section{débit de la turbine débit total}

Le mélange de l'eau turbinée et de l'eau en excès n'est assuré qu'à la partie périphérique de l'écoulement. Pour remédier à cet inconvénient Dauckwerts avait imaginé plusieurs tuyères coniques disposées concentriquement l'une derrière l'autre et de diamètres croissant dans le sens de l'écoulement.

Les renforceurs d'Herschelr conduisent à des cubes de maçonnerie très importants. Leur rendement n'est intéressant que pour des crues déjà notables. Ils n'excluent pas la diminution de la chute aux faibles crues qui, souvent, sont les plus fréquentes.

b) La tuyère de Fresmax. --- 'Toujours dans le but d'assurer un mélange plus efficace de
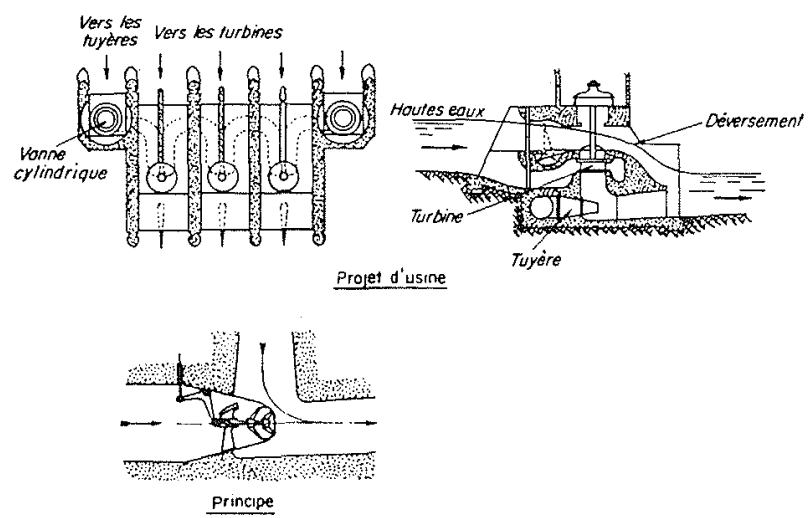

Fig. 2. - Tuyère Fireman.

deux veines, Freeman a proposé (1928) de pourvoir l'amont du diffuseur d'une tuyère destinéc à faire passer dans celui-ci, et à grande vitesse, l'eau non turbinée pour y accroître la dépression, et ainsi la hauteur de chute effective (fig. 2). Des aubes de guidage communiquent à l'eau un mouvement hélicoïdal et permettent ainsi un meilleur mélange avec l'eau turbinée. L'orifice de la tuyère est de dimension variable et mancuvrable par un levier commandé de l'extérieur.

Des essais effectués par Gribert ont montré que comparativement à d'autres dispositifs, la tuyère de Freeman provoque, toutes choses égales d'ailleurs, une dépression accrue du remous à l'aval de la turbine. La dépression produite dans le diffuseur accroìt légèrement le débit de la turbine. Mais, l'augmentation de Ia vitesse de l'eau turbinée, dans le difluseur, fait perdre à celui-ci une partie de son ròle qui était précisément d'augmenter l'énergie cédée à la turbine par récupération des vilesses.

La tuyère de Frisman de débit limité est surtout intéressante pour des crues moyennes et d'assez longue durée.

\section{2" Disposithes des Usines de Chèras e'T DU Mitchelldam.}

Ces dispositilis provoquent une dépression de la surface libre immédiatement à l'aval de la turbine.
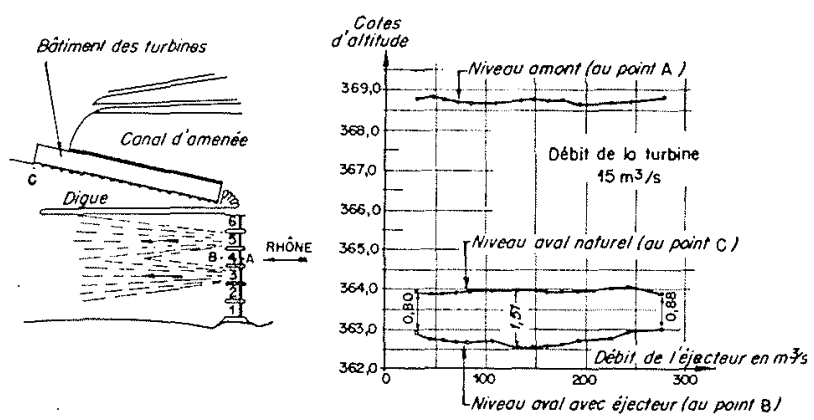

Fiti. 3. -..- Essais de Savgrix

a l'usine de Chèvers.

a) Usine de Chinres près de Genève.-... Essais de: SAUGEY (III) :

Savarr, pour l'usine de Chives, a utilisé les vannes du barrage de retenue comme éjecteur fruste, afin d'abaisser le plan d'eau à l'aval des lurbines. Il a effectué quelques essais intéressants en 1905 directement dans le Rhone. Notamment, il a fait jouer a un pertuis du bar-

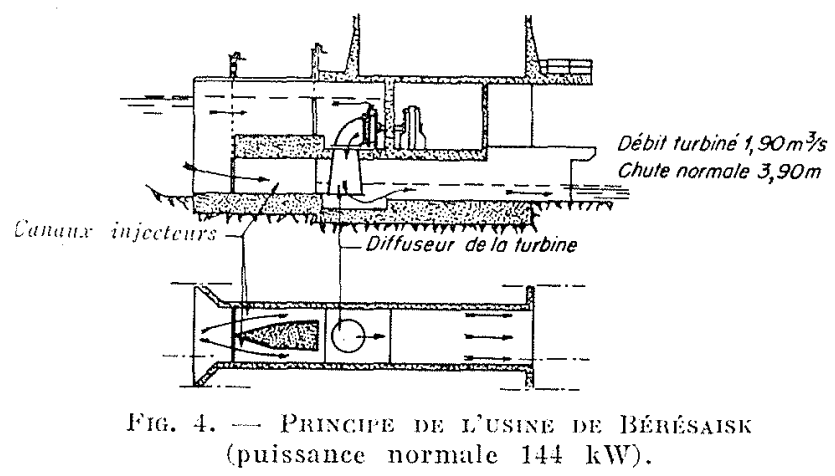

rage de retenue le ròle de turbine fictive, les pertuis latéraux servant de canaux injecteurs. II en résulte que le niveau aval est localement déprimé.

La figure 3 donne les résultats d'un essai dans les conditions suivantes : débil de la turbine fictive : $15 \mathrm{~m}$ " $/ \mathrm{s}$, débit de l'éjecteur, variable de 30 à $290 \mathrm{~m}^{3} / \mathrm{s}$. Le gain absolu sur la hauteur de 
chute passe par un maximum de $1,50 \mathrm{~m}$ environ; sa valeur pour les débits extrêmes est de 0,80 à $0,90 \mathrm{~m}$. Le gain relatif sur la hauteur de chute est de 15 a $30 \%$.

Des essais sur modele de Grubrirt montrent que la dépression de la surface libre se distribue d'une manière sensiblement uniforme dans toute la section. L'action unilatérale d'une vanne dans les mêmes conditions de débit total est également sans influence notable.

Dans le même ordre d'idće que les essais de SAUgey, Egoroff (IV) signale l'aménagement de petites usines russes par écoulement des caux en excès de chaque coté du dilluseur vertical, selon le dispositif de la figure 4 (Centrale de Bérésaïsk). Pour cette usine équipée de trois turbines de $82 \mathrm{ch}$, sous une chute de $3,90 \mathrm{~m}$ (débit de $1,87 \mathrm{~m}^{3} / \mathrm{s}$ ) la chute diminue de moitić pour la crue maximum $\left(160-180 \mathrm{~m}^{3} / \mathrm{s}\right)$. Les dispositions prévues permettraient :

- Pour la crue de $180 \mathrm{~m} 3 / \mathrm{s}$ de ramener la puissance théorique de la centrale de $144 \mathrm{~kW}$ tombée à $5.3 \mathrm{~kW}$, a la valeur de $88 \mathrm{~kW}$.

- Pour toute crue inférieure à $60 \mathrm{~m} \% / \mathrm{s}$ de maintenir la puissance normale.

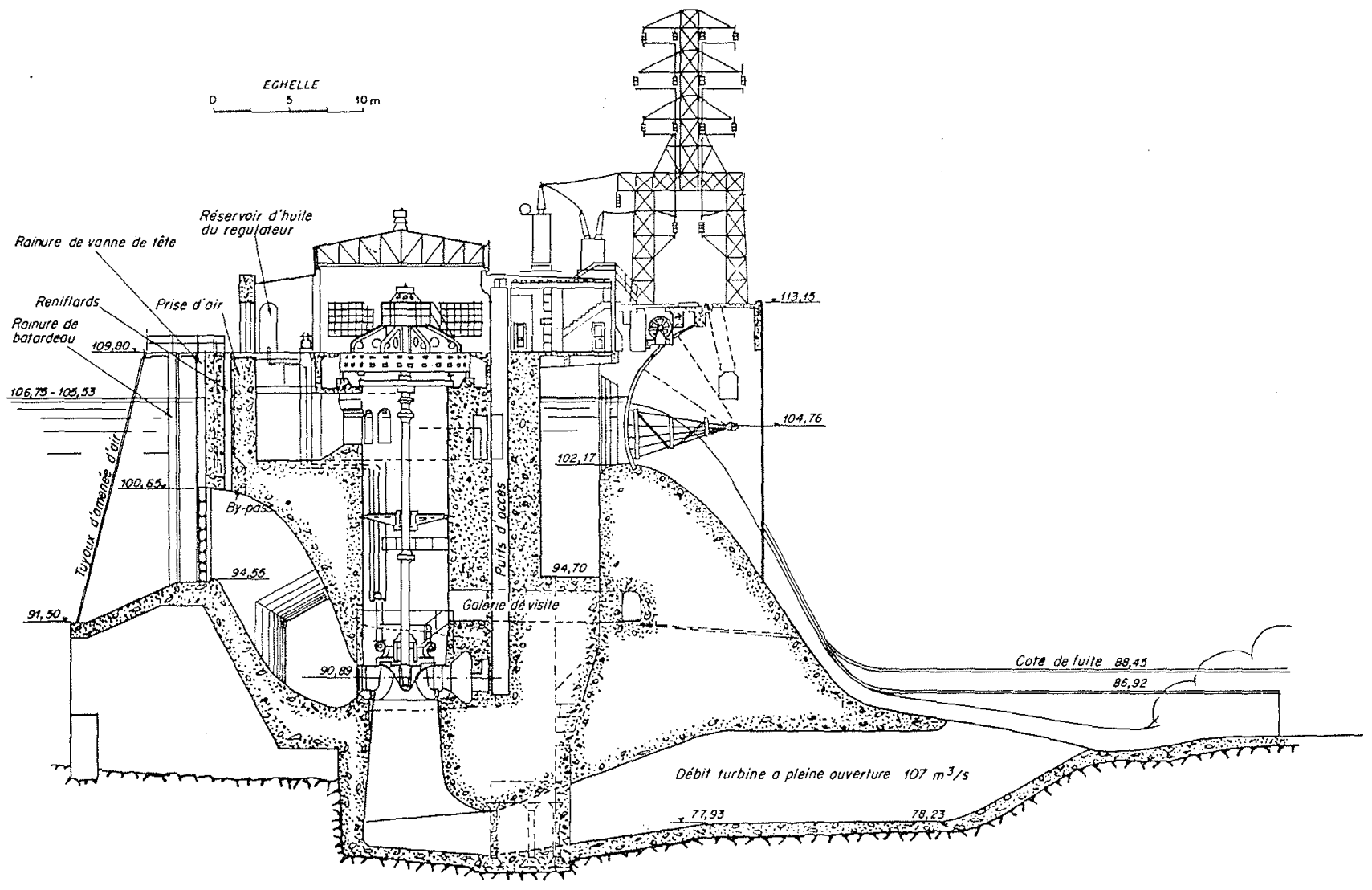

Fig. 5. - Using de barmage de Mrtinel. (1923).

\section{b) Usine du Mitchelldam :}

A la suite des essais d'injection de jets a grande vitesse dans les diffuseurs des turbines aux Etats-Unis dont les résultats nont pas été entièrement satisfaisants, on a cherché à provoquer une dépression du remous immédiatement à l'aval des diffuseurs des turbines.

A l'usine du Mitchelldam, on assure, par déversement des eaux en excès sur le barrage, la formation d'un ressaut a l'aval du diffuseur (fig. 5). Les essais effectués sur un modile au $1 / 10$ ont donné, par exemple, pour un type de jonction étudié, les résultats représentés figure 6.

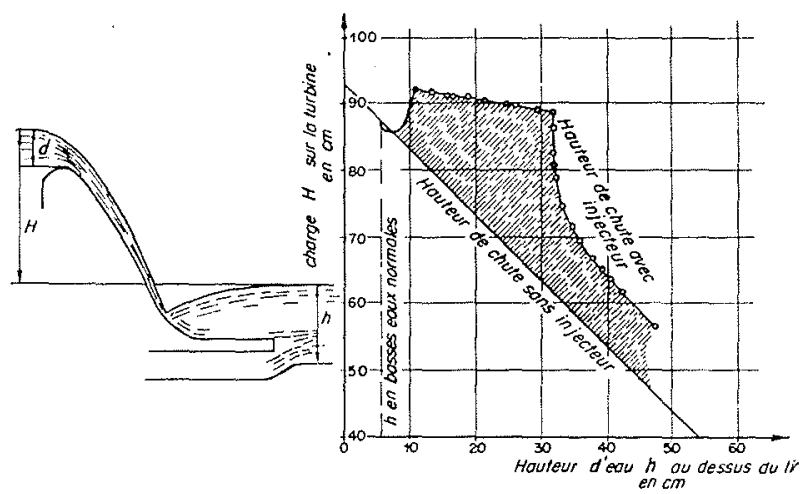

Fir. 6. - Usine de M[tchel. Dam. Essai sur modèle au $1 / 10$. 
Le gain relatif sur la hauteur de chute passe par un maximum et atteint pres de $30 \%$ de la hauteur de chute normale.

Les réalisations que nous avons examinées n’ont pas, en général, été renouvelées. Les frais de construction supplémentaire diminuaient notablement l'intérêt de ces dispositifs.

Pour les usines de types classiques, certains cas particuliers peuvent justifier la réalisation d'un renforceur de chute. Dans le cas de l'usine de Faux-la-Montagne, celui-ci existait implicitement et pouvait être réalisé correctement sans augmentation des frais de construction.

Le problème des usines piles et submersibles est plus complexe et nécessite initialement une étude détaillẻe, en particulier du phénomène d'échange de quantités de mouvement entre deux ou plusieurs veines de vitesses différentes.

\section{CHAPITRE II}

\section{ÉTUDE DE LA JONCTION DU CANAL DE FUITE ET DU CANAL DE DÉVERSEMENT DE L'USINE DE FAUX-LA-MONTAGNE}

\section{I. - Le problème}

On a vu que pour atténuer la remontée du plan. d'eau aval en période de crue et assurer un bon fonctionnement de l'usine automatique de Faux-la-Montagne, on avait été conduit à réunir le canal de déversement et le canal de fuite à une soixantaine de mètres à l'aval de l'usine.

L'étude sur modèle au $1 / 25$ de la jonction de ces deux canaux a porté sur les points suivants :

1" Maintenir aussi faible que possible le tirant d'eau dans le canal de fuite à l'usine, quelle quc soit la crue; ceci dans les conditions essentielles suivantes :

La cote du canal de fuite à radier horizontal, est fixée à $668,25 \mathrm{~m}$. Le canal de déversement au voisinage de la jonction a une pente de 15 à $18 \%$.

Le débit turbiné est de $8 \mathrm{~m}^{3} / \mathrm{s}$; la crue maximum est estimée à $20 \mathrm{~m}^{3} / \mathrm{s}$; il en résulte que, lorsque l'usine fonctionne, le débit dans le canal de déversement peut varier de 0 à $12 \mathrm{~m}^{3} / \mathrm{s}$.

2' La réunion de l'écoulement de fuite et de crue, dont les vitesses sont essentiellement différentes, doit s'effectuer dans des conditions hydrauliques acceptables et la restitution à la Maulde se faire en régime fluvial.

$3^{\circ}$ Pendant la construction ou réparation de l'usine, l'eau est évacuéc par le canal de déversement. Il convenait d'examiner si le niveau de l'eau qui, ainsi, pénètre dans le canal de fuite pouvait être suffisamment abaissé, pour supprimer le batardeau de l'usine, et le remplacer par de simples batardeaux en terre dans le canal do fuite.

La variation du plan d'eau de la Maulde où s'effectue la restitution est défini par la loi I approximative.

\begin{tabular}{|c|c|}
\hline $\begin{array}{c}\text { Débit restitue a } \\
\text { la Maulde }\end{array}$ & $\begin{array}{c}\text { Cote de } \\
\text { la Manlde }\end{array}$ \\
\hline & \\
\hline $10 \mathrm{~m} / \mathrm{s}$ & $668,70 \mathrm{~m}$ \\
$20 \mathrm{~m} / \mathrm{s}$ & $669, \mathrm{~s}$ \\
& $669,50 \mathrm{~m}$ \\
\hline
\end{tabular}

\section{II. - Principe et étude de la solution}

1. - En ce qui concerne lc canal de fuite, on recherche quel que soit le débit évacué par le canal de déversement à imposer à l'extrémité aval du canal de fuite le passage par le tirant d'eau critique.

En effet, si le tirant d'eau critique est obtenu à l'extrémité aval du canal de fuite, l'écoulement à la jonction même, n'a aucune influence sur l'écoulement dans ce canal. Le tirant d'eau at l'usine qui ne dépend plus, pour un débit donne, que des pertes de charge dans le canal, est maintenu constant et minimum. Pratiquement, on impose le tirant d'eau critique en un point du canal de fuite par décrochement brusque du radier, moyen parfaitement efficace si le niveau aval n'est pas excessif.

2. Pour le canal de déversement. il y a licu de rechercher le profil optimum de ce canal qui permette l'injection la plus efficace de la veinc de crue dans l'écoulement de fuite.

On dininue ainsi l'importance du tirant d'eau dans la jonclion à l'aval du canal de fuite, et 
facilite l'obtention de l'écoulement crilique à l'extrémité aval du canal de fuite.

On est ainsi conduit :

- Dune part, à limiter la dissipation d'énergie dans le canal de déversement avant la jonction, en diminuant l'importance des pertes par frottement sur les parois, par augmentation du rayon hydraulique et en évitant toute perte d'énergie causée par un elargissement trop brusque du canal de déversement avant la jonction.

Sur modèle, l'utilisation de parois mobiles permet de rechercher rapidement la position optimum.

- D'autre part, à faciliter l'échange des quantités de mouvement des deux veines à vitesses différentes. L'échange apparemment le plus favorable a été obtenu en cherchant à superposer les deux veines, la veine à grande vitesse se trouvant endessous.

On réalise approximativement la superposition des deux veines en fixant le radier du canal de déversement à une cote légèrement inférieure à l'extrémité aval du canal de fuite.

3. - La jonction a un double but à remplir :

a) Sa forme, particulièrement en plan, doit être telle que l'écoulement s'effectue avec le
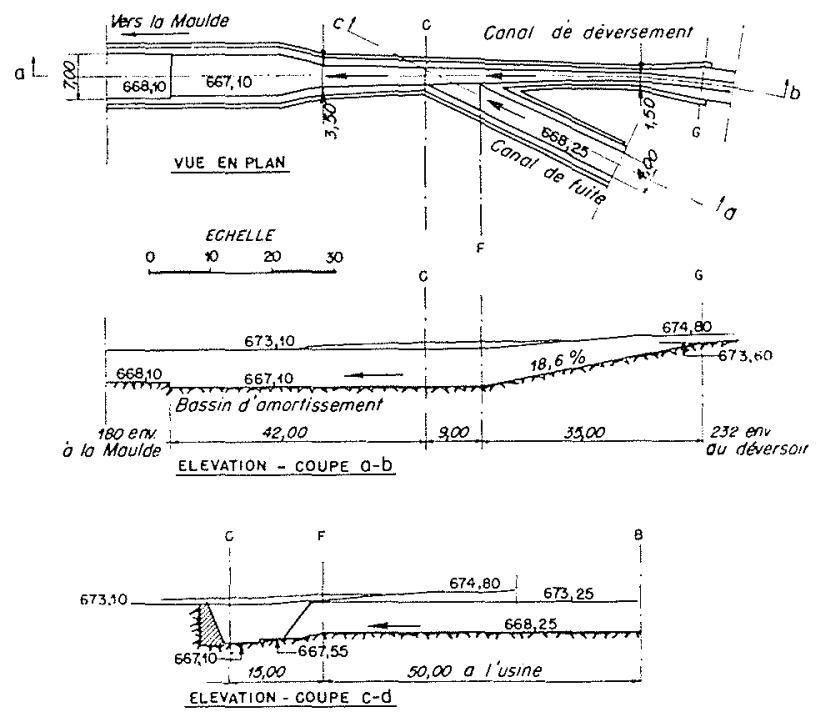

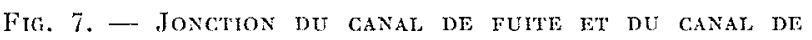
DÉVERSEMENT. SOLUTION ADOp'TÉ.

minimum de perturbations et ainsi ne provoque pas de surélévation exagérée du plan d'eau dans le canal de fuite.

L’inégale répartition des vitesses dans une section du canal de déversement due a la courbure en plan du canal, produit à la jonction des tourbillons à axes verticaux dont le déplacement en rouleaux s'oppose à l'écoulement du débit turbiné. On constate sur modèle que la formation de ce courant de retour est très atténué lorsque le raccordement en plan de la rive gauche du canal de fuite à la jonction est réalisée par un angle vif et non par une courbe de grand rayon.

b) Elle doit également assurer la localisation du ressaut qui marque le passage de l'écoulement torrentiel de crue à l'écoulement fluvial.

Un bassin d'amortissement de dimensions acceptables permet d'assurer la formation d'un ressant stable lorsque la crue exceptionnelle de $20 \mathrm{~m}^{3} / \mathrm{s}$ passe entièrement par le canal de déversement, l'usine étant arrètée.

L'étude sur modèle de ces différents principes conduit à la solution définitive (fig. 7) qui donne la plus forte dépression à l'aval du canal de fuite.

- Le radier horizontal du canal de fuite est maintenu à la cote 668,25 , sauf à l'extrémité aval qui se présente sous l'aspect d'un double décrochement; celui-ci, d'une part, permet d'imposer le tirant d'eau critique sauf aux faibles crues, et d'autre part facilite, à la jonction, la superposition des deux veines liquides.

- Le canal de déversement s’élargit progressivement de 1,50 à $3,50 \mathrm{~m}$ sur une longueur de 50 mètres; sa pente est roisine de $18,6 \%$.

- La jonction sert de bassin d'amortissement; la longueur et la profondeur en sont minimum. La cote supérieure du seuil aval est fixée à $669,10 \mathrm{~m}$.

\section{III. - Les résultats obtenus}

Compte tenu de la loi I de variation du plan d'eau de la Maulde au voisinage de la restitution, précédemment énoncée, les essais ont mis en éridence les résultats suivants :

1. - Variation du tirant d'eau dans le canal de fuite à l'usine, lorsque l'usine fonctionne normałement, en fonction du débit $q_{c}$ d'eau en en excès, évacué par le canal de déversement.

Le tirant d'eaü dans le canal de fuite à l'usine passe par un maximum pour un débit de crue voisin de $4 \mathrm{~m}^{3} / \mathrm{s}$. L'amplitude de cette variation est de $0,19 \mathrm{~m}$. Il est intéressant de comparer cette courbe (fig. 8, courbe 1):

- D'une part, à la courbe (2) tracée lors d'une étude préliminaire légèrement différente de la solution définitive. On roit ainsi l'intérêt d'étudier avec soin les meilleures conditions d'injection et d'écoulement. 
Tiront d'eau

du conol de fuite o l'usine

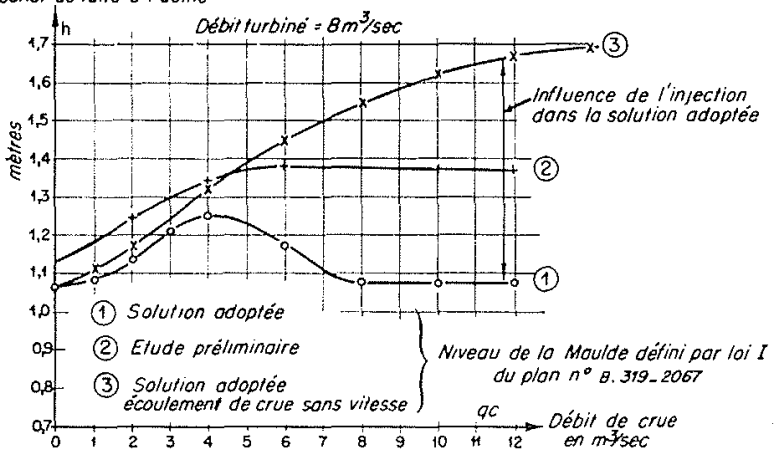

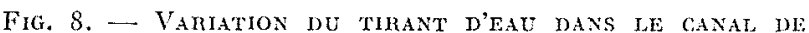
FUITE A LuSINE EN FONCTION DU DÉBIT DE CRUE POUn DHFERENTES FORMES Dl: rA JONCTION.

- D'autre part, à la courbe (3) dans l'hypothèse où le débit évacué par le canal de déversement est supposé arriver à la jonction pratiquement sans vitesse. La diflerence des ordonnées des courbes (1) ot

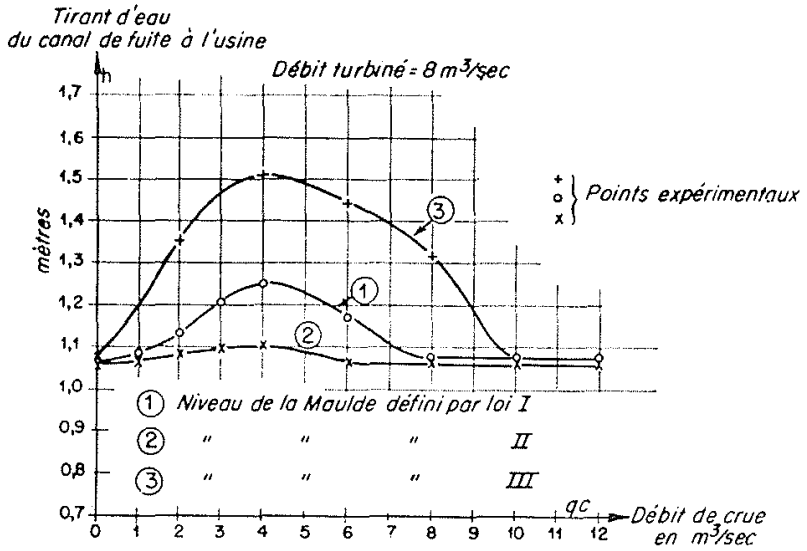

Fig. 9. --. Variation du trmant d'tay dans le canal, de FUTE A l'USINE EN FONCTION DU DÉMT DE CRUT,

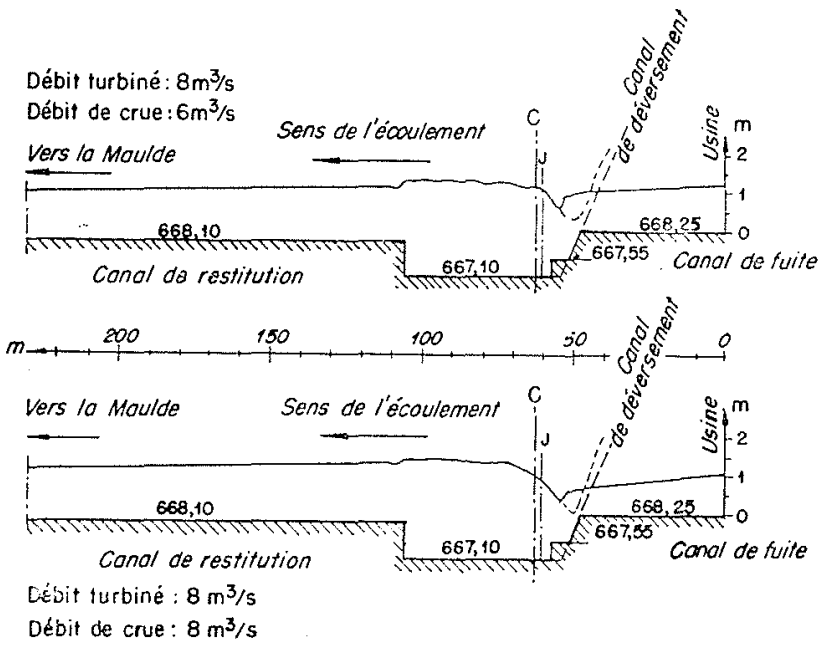

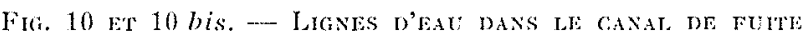
IT A la roxctrox.
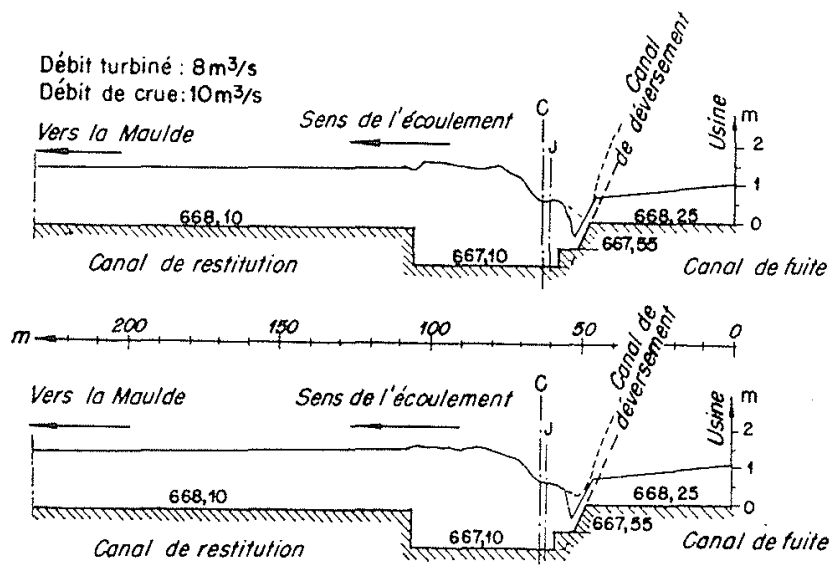

Débit turbiné : $8 \mathrm{~m}^{3} / \mathrm{s}$

Débit de crue : $12 \mathrm{~m}^{3} / \mathrm{s}$

(3) met en évidence l'action de l'ejecteur réalisé.

On peut rechercher l'influence de la variation du plan d'eau de la Maulde en période de crue. La loi I admise, présente certainement une sécurité. Ta loi II ci-dessous :

\begin{tabular}{|c|c|}
\hline Débil restitué & Niveau de la Maulde \\
\hline & \\
\hline $8 \mathrm{~m}^{3} / \mathrm{s}$ & $668,40 \mathrm{~m}$ \\
$10 \mathrm{~m}^{3} / \mathrm{s}$ & $668,70 \mathrm{~m}$ \\
$20 \mathrm{~m}^{3} / \mathrm{s}$ & $669,20 \mathrm{~m}$ \\
\hline
\end{tabular}

qui fait correspondre au débit restitué une cote inférieure de la Maulde, conduit pour la variation du tirant d'eau dans lo canal de fuite a l'usine, a la courbe (2z) de la figure 9. La courbe (3) est relative à une loi encore plus pessimiste que la loi I. On voit ainsi que, pratiquement, Ic

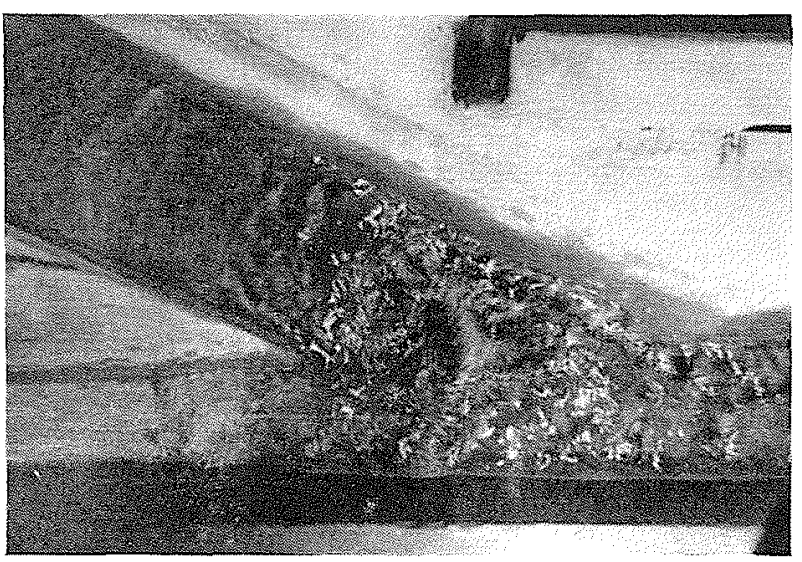

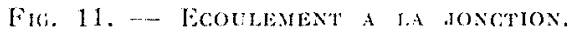
Débit turbiné : $8 \mathrm{~m} 3 / \mathrm{s}$. - Débit de crue $: 4 \mathrm{~m}: \mathrm{s}$. 
dispositif adopté permet de maintenir constante à quelques centimètres près, la hauteur de chute de l'usine.

2. - Les lignes d'cau dans le canal de fuite et à la jonction font apparaître l'cfret de l'injection, notable à partir d'un débit de $5 \mathrm{~m}^{3} / \mathrm{s}$ dans le canal de déversement (fig. 10).

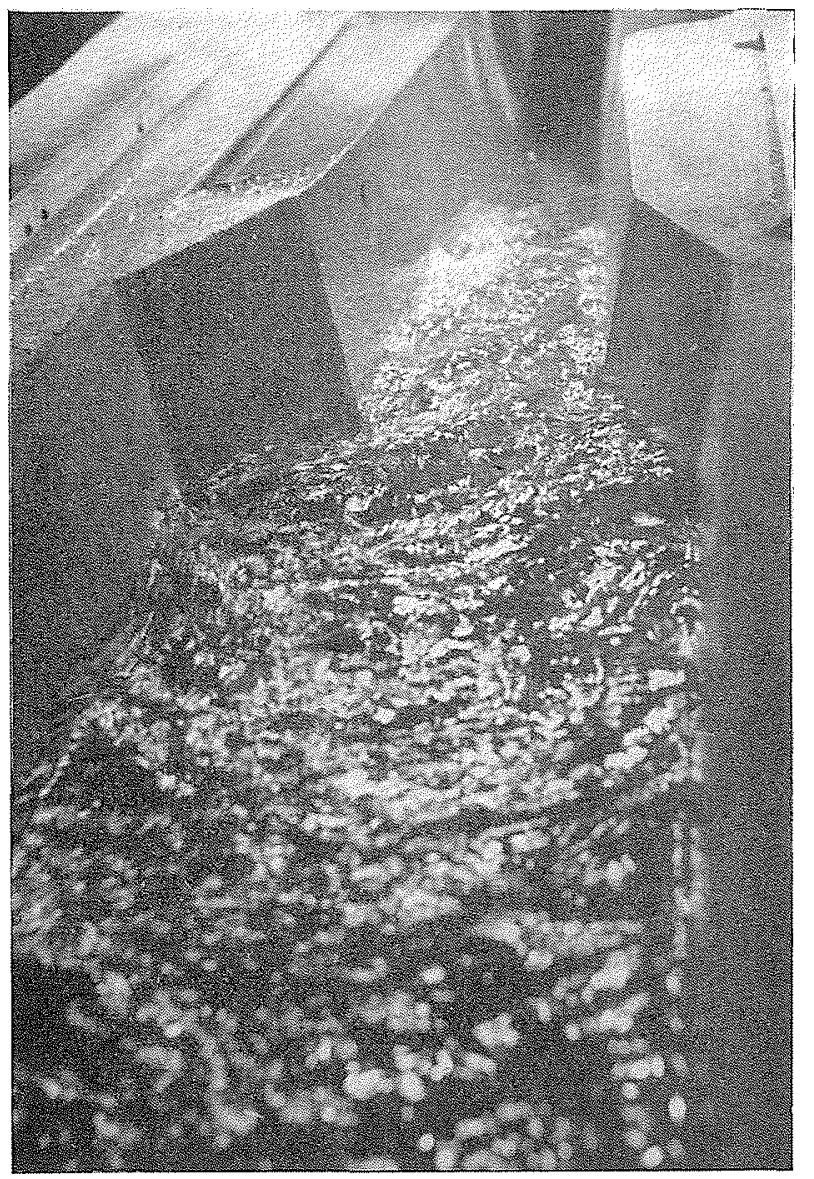

Fig. 12. - Ecoulement dans Le Bassin D'AMORTSSEMENT. Débit turbiné : $8 \mathrm{~m}^{3} / \mathrm{s}$. - Débit de crue : $8 \mathrm{~m}^{3} / \mathrm{s}$.

3. - Lorsque l'usine ne fonctionne pas et que l'eau passe exclusivement dans le canal de déversement, le plan d'eau dans le canal de fuite atteint au maximum $38 \mathrm{~cm}$ au-dessus du radier. On voit qu'il est possible de supprimer le batardeau de l'usine. Pour des crues supérieures à $6 \mathrm{~m}^{3} / \mathrm{s}$, il n'y a plus d'eau dans le canal de fuite.

Les photographies $n^{\text {os }} 11-12-13$ et 14 donnent l'aspect de l'écoulement, pour différents débits du canal de déversement et compte tenu de la loi I du niveau de la Maulde.

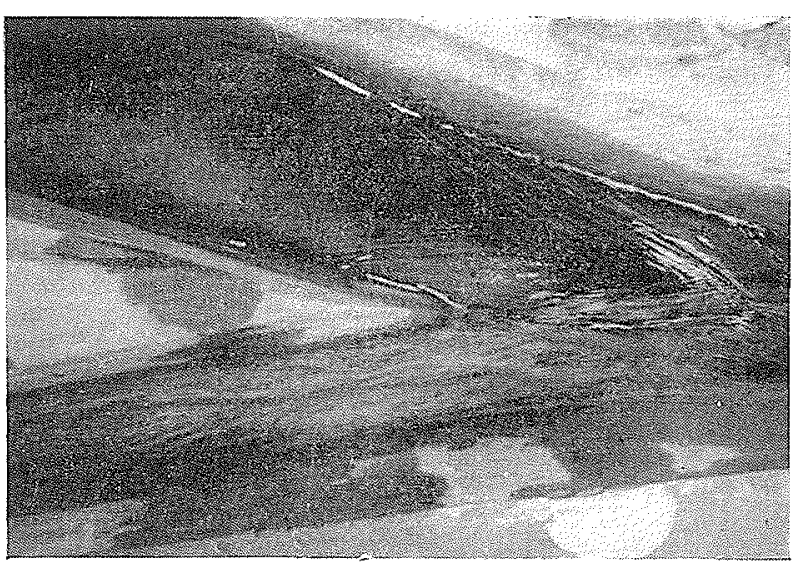

Fig. 13. - Ecoulement a la ronction.

Dẻbit turbiné : $8 \mathrm{~m}^{3} / \mathrm{s}$. - Débit de crue : $10 \mathrm{~m} 3 / \mathrm{s}$.

\section{IV. - Conclusions}

L'étude sur modèle au $1 / 25$ de la jonction du canal de déversement et du canal de fuite a permis de réaliser, sans augmentation des frais de construction, un dispositif permettant de maintenir très sensiblement constante, en période de crue, la hauteur de chute de l'usine.

Le gain relatif de ce dispositif, dans le cas d'espèce d'une usine de moyenne chute, est nécessairement faible. Mais cette étude laisse supposer que dans des cas bien adaptés de basse chute, la réalisation de renforceurs de chute puisse être justifiće. A ce sujet, nous allons examiner quelques nouvelles conceptions dans la construction d'usines de basses chutes.

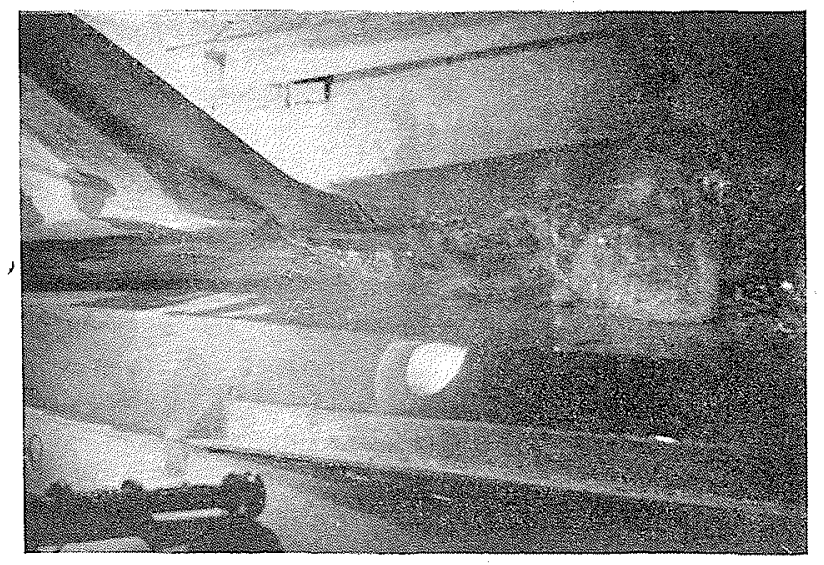

Fig. 14. - Ecoulement a la JONCTion.

Débit turbiné : $8 \mathrm{~m}^{3} / \mathrm{s}$. - Débit de crue : $12 \mathrm{~m} 3 / \mathrm{s}$. 


\section{LES NOUVELLES CONCEPTIONS DES USINES DE BASSE CHUTE}

En examinant les nouvelles conceptions qui se sont développées pour la construction des usines de rivières, notre but est simplement ici de faire apparaître brièvement. Ies possibilités' d'exploitations de ces usines en hautes eaux.

\section{1. - Les usines piles}

SAUgey proposait en 1905 de fractionner les usines de rivières construites en un seul bloc près d'une rive, et de répartir les groupes de machines dans l'ensemble de l'ouvrage de retenue, en alternant avec les pertuis du barrage mobile. Son idée directrice était de récupérer une partie de la chute perdue en hautes eaux. Ce projet n'eut aucun succès à l'époque par suite de l'augmentation continuelle de la hauteur de chute des usines de rivières, au cours des années suivantes.

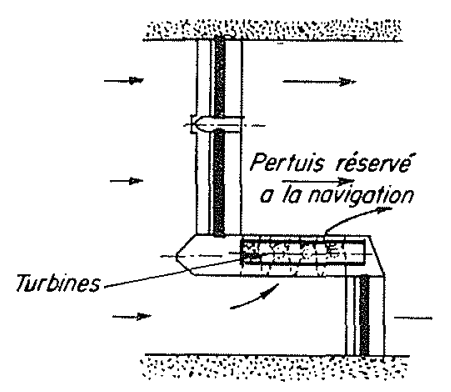

Usines piles sur le Moin

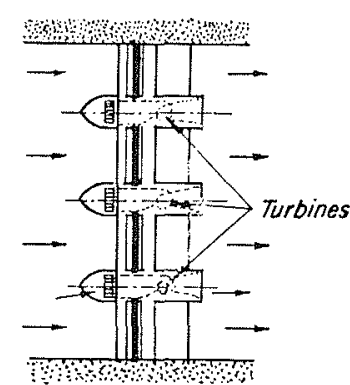

Usines piles sur lo Drove
FIG. 15. - SCHÉMA DE PRINCIPE DES USINES-PILES.

Quelques réalisations particulières furent entreprises pendant la première guerre mondiale, sur le Main : usines de Krotzenburg, Kesselstadt, Mainkur. Les centrales se présentaient sous forme d'une pile comprenant tous les groupes, et implantée parallèlement à l'écoulement (fig. 15) (VI).

Dans les quiñze dernières années la conception d'usines piles a été reprise à la faveur des arguments suivants : diminution de la longueur totale de l'ouvrage de retenue par suppression des piles du barrage, - amélioration de l'aspect extérieur de l'ouvrage, - répartition plus uniforme du débit entre les turbines et à l'aval du barrage, - possibilité de récupérer une partie de la chute perdue en hautes eaux. Les principales réalisations furent effectuées sur la Drave (Usine d'Unter-Drauburg, Usine de Marburg).

En ce qui concerne la récupération d'une partie de la chute perdue en hautes eaux, les usines piles permettent de concevoir les solutions suivantes :

- Utilisation des pertuis entre piles comme injecteurs de part et d'autre des turbines (idée de SaUgey).
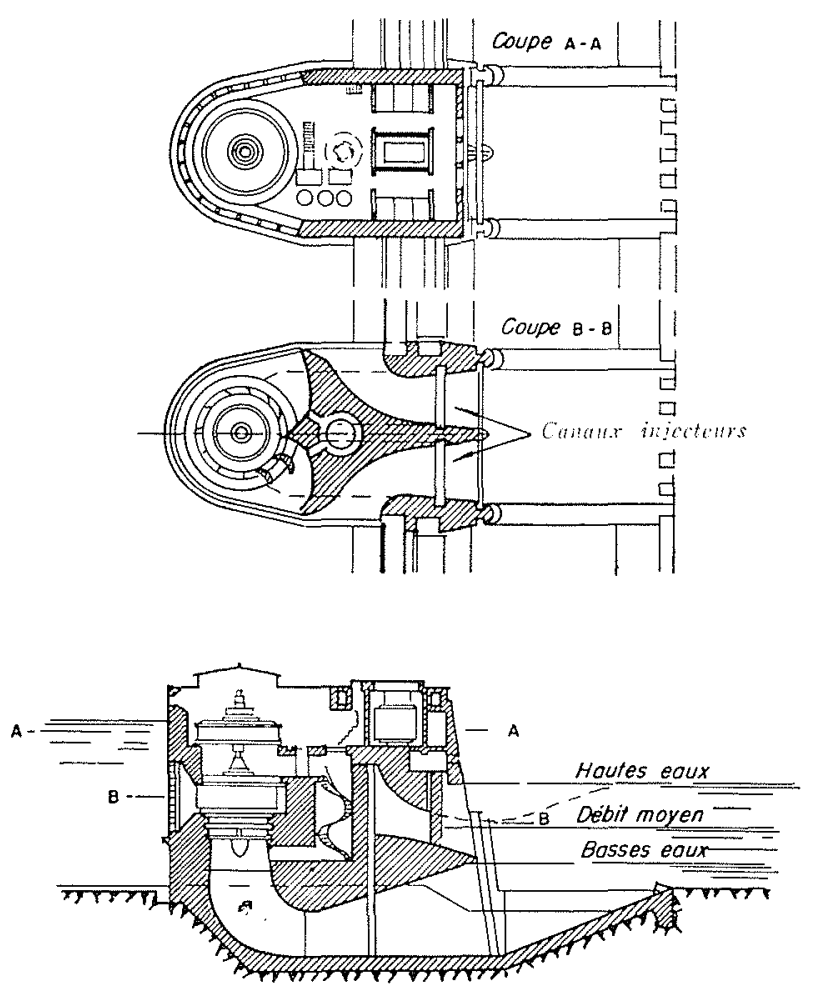

Fig. 16. - Projet D'usines-riles d'après B. Schultz.

- Réalisation dans la pile même de canaux injecteurs au-dessus du diffuseur de la turbine (projet de ScHulz). L'effet d'injection est obtenu par la superposition des deux écoulements; l'écoulement à grande vitesse se trouve à la partie supérieure (fig. 16) (VII). 
- Réalisation d'un canal injecteur sous le diffuseur de la turbine. Il y a superposition des deux écoulements mais l'écoulement à grande vitesse se trouve cette fois à la partie inférieure.

- Mise en place de déflecteurs sous les volets de déversement pour concentrer l'énergie des eaux en excès prés des sorties des turbines.

\section{II. - Les usines submersibles}

Le développement des usines submersibles a été surtout lié à la réalisation d'un nouveau type
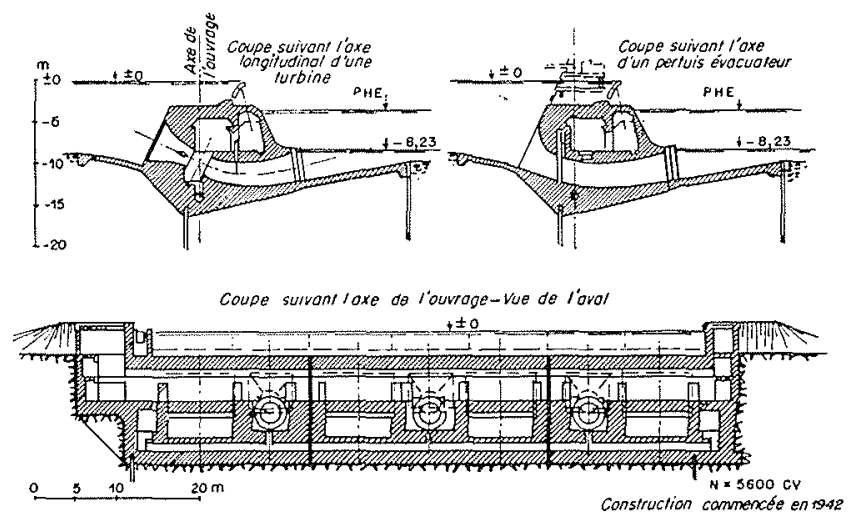

Fic. $17 a$. - Centrale submisrible de Freilassing. Sur la SaAlach (Bavière-Autriche).

(Extrait du projet de novembre 1941.)
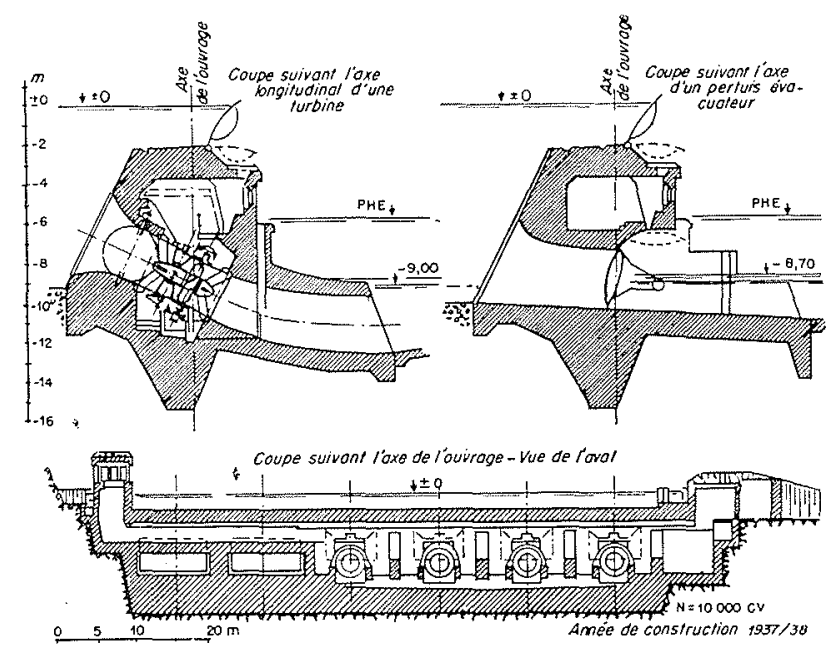

Fig. 17 b. - Centrale submersible de Steinbach Sur L'Illerr (Bavière).

(Extrait du projet d'avril 1937.) de turbine à axe incliné de dimensions plus réduites que les turbines du type classique et généralement connue sous le nom de turbine A. Fisciner.

La répartition des groupes est effectuée dans l'ensemble de l'ourrage qui barre complètement la rivière. La crue est évacuée en partie par des chasses situées en général entre les turbines (Usine de Rostin sur la Persante, Usine de Freilassing sur la Saalach...) exceptionnellement sur un côté de l'usine (Usine de Steinbach sur l'Iller) et par déversement sur l'ensemble de l'ouvrage. Les chasses entre les turbines jouent le rôle de canaux injecteurs et devaient permettre dans l'esprit des constructeurs une récupération d'énergie de $30 \%$ pendant les hautes eaux (fig. 17) (IX).

Les résultats de l'exploitation de ces usines pendant les périodes de crue ne sont pas publiés. On pense que le gain serait de 10 à $12 \%$.

Les usines submersibles permettent de concevoir le maintien de la chute en période de hautes eaux par des dispositíts semblables à ceux des usines piles.

L'intéret des renforceurs de chute et l'importance qu'il convient de leur donner sont essentiellement dominés par une question économique. Certains dispositifs coûteux (par excmple canal injecteur sous le diffuseur de la turbine) ne peuvent se justifier que par un bon rendement.

Une étude systématique de laboratoire relative, d'une part, à l'échange des quantités de mouverneni entre écoulements à vitesses différentes, et, d'autre part aux dispositifs pratiques utilisables pour les centrales hydro-électriques est à la base de toute conception rationnelle d'usine de basse chute. Cette étude est actuellement abordée au Laboratoire National d'Hydraulique à Chatou.

\section{BIBLIOGRAPHIE}

(1) R. Gelbert. - Gefälvermehrung bei NiederdruclWasserkraftanlagen. Augmentation de la hauteur de chute des usines de basse chute. Berlin, 1930. Verlag von Wilhelm Ernst und Sohn.

(II) D. Exdoux. - Hydraulique industrielle et usines hydrauliques. Paris. J.-B. Baillière et Fils, 192, pp. $418-420$.

(III) Foch. - Utilisation des crues pour renforcer les chutes. La Houille Blanche, mars-juillet 1923.

(IV) Egonofr. - Renforceur de chute pour petites centrales hydrauliques. Constructions Hydrotechniques U.R.S.S., 1949, n 8 , pp. 20-25. 
(V) $\mathrm{X}^{\star * *}$. - Usinc-barrage Mitchell (1923): Essai de compensation de la perte de chute en hautes eaux. Electricité de France. Traduction. Février 1950 .

(VI) Stankach. - Les centrales aménagées dans les piles de barrage hier et aujourdhui. Schweizerische Bauzeitung, 5 juillet 1947 .
(VII) Schutz. - Eine neate Pleilertraftwerksbanart. Un nouveau type d'usine hydro-électrique: l'usinepile. Die Wasserwirtschaft, $\mathrm{n}^{\circ}$ 2, 1949-1950.

(VIII) - Centrales hydro-électriques submersibles, type A. Fischer. Rapport Electricité de France.

(IX) H. Frntzloff. - Principes fondamentaux de la construction des centrales submersibles. La Houille Blanche, septembre-octobre 1949.

\section{COMMENTAIRE DE M. LE PRÉSIDENT}

Je remereic M. Marouenet, pour la clarté de son exposé, et aussi pour la façon précise avec laquelle il s'est conformé au programme qui lui avait été imposé.

Naturellement, M. Marouener aurait pu nous donner beaucoup d'autres détails, mais finalement, dans le temps que nous lui avons consacré, il a réussi à dire tout ce qu'il avait à dire. Ie le félicite, par conséquent, pour la bonne proportion qu'il y a entre les moyens utilisés et les résultats obtenus.
D'autre part, l'etude nous laisse entendre pour plus tard des compléments. Par conséquent, je crois qu'il n'y a pas lieu de faire nue très grande discussion sur ce mémoire, dont les deux parties sont nettement séparées : l'une historique, qui rappelle ce qui a été fait antéricurement, et la deuxième qui est relative aux essais qui ont été faits à Chatou. La troisième partie viendra ultérieurement, par conséquent.

Je remercie M. Marquenet, et je souhaite de le revoir it une ultérieure session.

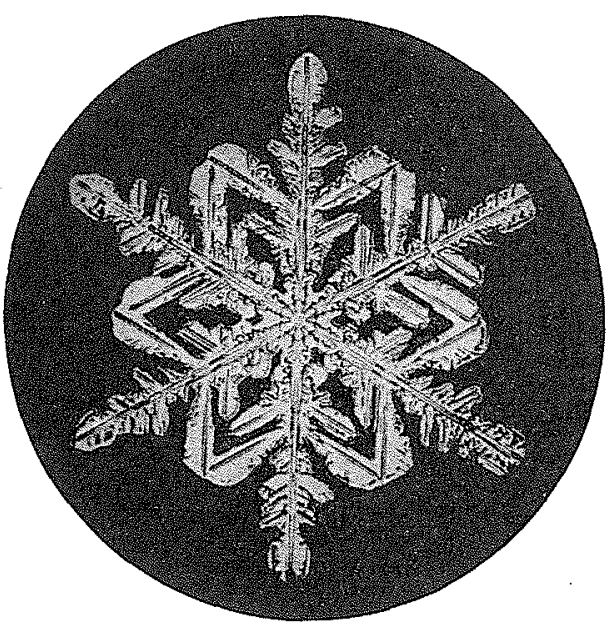

